\title{
Classical Peer Review
}

Richard Walker, Pascal Rocha da Silva

\section{Source}

Richard Walker, Pascal Rocha da Silva. (2015). Emerging trends in peer review-a survey.

Front. Neurosci., vol. 9.

We define classical peer review as a process which:

- Assesses the suitability of a manuscript for publication, and provides feedback to

authors, helping them to improve the quality of their manuscripts

- Follows formal procedures and assessment criteria

- Takes place before publication

- Is highly selective

- Assesses manuscripts in terms of their novelty, "importance for the field" and "interest for a broad readership"

- Is conducted by a small number of editor-selected expert reviewers, whose names are not revealed to authors or readers, and who make their assessments without any direct interaction among themselves or with authors

- Concludes with a publication decision taken by the editor(s). 